\title{
Comparison and suitability of genotype by environment analysis methods for yield-related traits of pearl millet
}

\author{
G. Lubadde', J. Ebiyau', B. Akello ${ }^{2}$ and M.A. Ugen ${ }^{1}$ \\ ${ }^{1}$ National Semi-Arid Resources Research Institute, P. O. Box 56, Soroti, Uganda \\ ${ }^{2}$ Mukono Zonal Agricultural Research and Development Institute, P. O. Box 164, Mukono, Uganda
}

Author for correspondence: glubadde@gmail.com

\begin{abstract}
Pearl millet (Pennisetum glaucum (L.) R. Br.) is an important food security and income crop for households living in semi-arid zones in Uganda. However, the genotype by environment interaction, in addition to the several methods used for its assessment, complicates selection of varieties adapted to such semi-arid areas. The objective of this study, therefore, was to compare common methods used to assess stability and adaptability of improved genotypes. Seventy six genotypes were planted in four environments in an alpha experimental design with two replications. Results showed that genotype by environment interactions were significant at $\mathbf{p}<0.05$ for grain yield, days to $50 \%$ flowering and $50 \%$ physiological maturity, percentage of productive tillers and panicle area. Results further showed inconsistency in ranking of genotypes between methods; although Cultivar Superiority, REML, Yield Stability Index and GGE biplot were consistently correlated and identified high yielding and stable genotypes.
\end{abstract}

Key words: GGE biplot, grain yield, pearl millet, stability analysis, Uganda

\section{Introduction}

Pearl millet (Pennisetum glaucum (L.) $\mathrm{R}$. Br.) is one of the widely grown millets with several food and non-food uses (IFAD, 1999). The crop responds positively to adverse environments that are extremely variable and often associated with erratic and low annual rainfall (Bashir et al., 2014). Despite the adaptability, average productivity of about $600 \mathrm{~kg} \mathrm{ha}^{-1}$
(Rai et al., 1999) from farmers' fields is low much as relatively high yielding genotypes adapted to low-input and drought-prone environments have been developed (Serraj et al., 2003; Vadez et al., 2012). This is partly because the potential performance of the high-yielding genotypes under marginalised conditions is always obscured by the multiplicative effect of genotype by environment interaction (GEI) (Yan and Racjan, 2002). 
Accordingly, this causes inconsistent performance of genotypes (Alberts, 2004), and thus leading to false selection (Crossa, 1990; Falconer, 1990).

It is in response to these challenges that it is necessary to assess genotypes for adaptability and stability (Becker and Léon, 1988). Equally important is the need to develop appropriate statistical models that have the rigor and accuracy to support selection decisions in case significant GEI exists, and hence identification of a reliable method is important (Yau, 1991).

Several statistical analysis methods have been developed to assess GEI, notable of which are; analysis of variance (ANOVA), environmental variance $\left(\mathrm{S}^{2} \mathrm{i}\right)$, deviation from regression $\left(\mathrm{S}_{\mathrm{d}}{ }_{\mathrm{d}}\right)$, Restricted Maximum Likelihood (REML) (Bartlett, 1937), regression coefficient $\left(b_{i}\right)$ (Finlay and Wilkinson, 1963), Wricke's ecovalence (Wi), Eberhart and Russell (1966), Best Linear Unbiased Predictions (BLUP) (Patterson and Thompson, 1971), Tai's (1971) approach, Shukla stability variance $\left(\sigma \dot{i}^{2}\right)$ (Shukla, 1972), coefficient of determination $\left(\mathrm{r}_{\mathrm{i}}^{2}\right)$ (Pinthus, 1973), coefficient of variation (CV) (Francis and Kannenberg, 1978), cultivar superiority $\left(\mathrm{P}_{\mathrm{i}}\right)$ (Lin and Binns, 1988) and static stability (Becker and Léon, 1988). Some of the most frequently used methods include; Additive Main Effects and Multiplicative Interaction (AMMI) (Gauch, 1988), yield stability index (YSi) (Kang, 1993), AMMI stability value (ASVi) (Purchase, 2000), Genotype and Genotype by Environment (GGE) biplot (Yan and Hunt, 2002) and harmonic mean of the relative performance of genotypic values (MHPRVG) (Resende, 2007). However, most of the methods have deficiencies.

The ANOVA identifies sources of variation due to GEI effect and allows for estimation of variance components used to calculate trait heritability. However, it does not explore the underlying structure within the GEI; making it difficult to establish the true performance of genotypes across environments (Crossa, 1990). The regression approach is widely used (Westcott, 1986; Freeman and Perkins, 1971) but limited in functionality because genotype response to environments is largely under multivariate control; yet regression transforms it into a univariate variable (Lin et al., 1986). Crossa (1990) also noted that parameters of regression (mean, slope, and deviation) also make it difficult to identify superior genotypes for particular environments. The YSi has a weakness of weighing strongly on yield, yet the trait is influenced by many factors (Farshadfar et al., 2011). Wricke's partition of the interaction is nonorthogonal yet the test is parametric (Freeman and Perkins, 1971). The AMMI models (Gauch, 2006; Gauch et al., 2008) combine the ANOVA for the genotype and environment main effects with principal components analysis which helps to obtain further insight into the nature and extent of complex GEI (Alberts, 2004; Gruneberg et al., 2005). However, there is difficulty in interpretation of the interaction when there is limited variability accounted for by the first principal component, which could indicate false statistical stability of the genotypes and environments (Lavoranti et al., 2007). The AMMI and the GGE biplot combine genotype (G) and genotype by environment (GE) in mega environment evaluation, but the GGE biplot is superior to the AMMI in graphical analysis because it better explains G+GE (Yan et al., 2007). The inadequacy and contrasting argument about the best stability and adaptability analysis methods of GEI shows that most 
probably no stand-alone method exists (Kaya et al., 2006). Thus the objective was to assess stability analysis methods for correlation and consistency using traits of improved pearl millet genotypes.

\section{Materials and methods}

\section{Test environments and materials}

The study was conducted for two rainy seasons which coincided with the second rains of 2012 and first rains of 2013. The evaluation was done in two locations (Kitgum and Serere) and this resulted in four environments. The Kitgum environments (E1 and E2) are located at $03^{\circ} 132 \mathrm{~N}, 032^{\circ} 472 \mathrm{E}, 969$ m.a.s.l while the Serere (E3 and E4) environments are located at $01^{\circ} 32^{\prime} \mathrm{N}, 033^{\circ} 27^{\prime} \mathrm{E}, 1140$ m.a.s.l. E1 received $391 \mathrm{~mm}$ of rainfall in 2012; while E2 received $817 \mathrm{~mm}$ of rainfall in 2013. E3 received $499.3 \mathrm{~mm}$ of rainfall in 2012; while E4 received $589 \mathrm{~mm}$ of rainfall in 2013). The environments were characterised as hot spots for rust disease (Lubadde et al., 2014), sandy soils and being semi-arid.

The 76 improved pearl millet genotypes evaluated were replicated twice in a $4 \mathrm{x}$ 19 alpha experimental design. The materials were planted in $8 \mathrm{~m} \times 5 \mathrm{~m}$ plots at a spacing of $60 \mathrm{~cm} \times 30 \mathrm{~cm}$. A soil fertility regime recommended for seed production under rain fed conditions was adopted and standard agronomic practices for crop management were used (Khairwal et al., 2007).

\section{Data collection and analysis}

Data were collected on at least 36 randomly selected plants per plot, using the 'Descriptors of Pearl Millet' (IBPGR and ICRISAT, 1993). The panicle area (PAR) was calculated as $3.14 \times \mathrm{L}$ x W; where $\mathrm{L}$ and $\mathrm{W}$ were panicle length and width, respectively. Data were also collected on: grain yield ( $\mathrm{GY}$ in $\mathrm{kg} \mathrm{ha}^{-1}$ ) at $50 \%$ physiological maturity after threshing, days to $50 \%$ flowering $\left(\mathrm{FLO}_{50}\right)$ at plot level when $50 \%$ of the plants have developed stigmas, days to $50 \%$ physiological maturity $\left(\mathrm{PSM}_{50}\right)$ and percentage of productive tillers (PRO) at plot level. Data analysis was conducted using the Integrated Breeding Platform for Breeding Management System version 3.0.8 (IBP-BMS, 2014) and GenStat $15^{\text {th }}$ Edition (Payne et al., 2012). The performance and ranking of genotypes was used to compare the consistency of the GEI methods. The models and computations for ANOVA, REML and AMMI indices for calculating ASVi were computed using GenStat 15 while the YSi, Wricke's ecovalence, Finlay and Wilkinson, static stability, cultivar superiority and were computed using IBPBMS 3.0.8.

\section{Results}

Assessing GEI effect using stability indices The ANOVA showed that the main effects of environments were significant $(\mathrm{p}<$ 0.05 ) on GY and $\operatorname{PSM}_{50}$ and highly significant $(\mathrm{p}<0.001)$ for FLO50, PAR and PRO. The main effects of the genotypes were also significantly $(\mathrm{p}<0.05)$ important for the yield-related traits except PAR. In addition, (GEI) was significant $(\mathrm{p}<0.05)$ for all the test traits.

Results for stability and GEI assessment for twenty most stable genotypes are shown in Tables 1-8. Generally, Cultivar superiority, REML, Yield stability index (YSi) and GGE biplot identified highly performing genotypes, as being stable with a significant positive correlation observed for most traits (Table 1) and among the methods (Table 2). A 
Table 1. Correlation between highly correlated stability methods and traits

\begin{tabular}{lcccc}
\hline Traits & Pi+REML & Pi+GGEbiplot & Pi+YSi & REML+YSi \\
\hline GY & $0.9^{* *}$ & $0.5^{*}$ & $0.5^{*}$ & $0.5^{*}$ \\
FLO $_{50}$ & $-0.8^{* *}$ & $0.5^{*}$ & $-0.5^{*}$ & $0.5^{*}$ \\
PSM $_{50}$ & $-0.9^{* *}$ & $0.5^{*}$ & $-0.6^{*}$ & $0.6^{* *}$ \\
PRO $_{\text {PAR }}$ & $0.9^{* *}$ & $-0.0 \mathrm{~ns}$ & $0.8^{* *}$ & $0.7^{* *}$ \\
& $0.6^{*}$ & $-0.0 \mathrm{~ns}$ & $0.1 \mathrm{~ns}$ & $-0.1 \mathrm{~ns}$ \\
\hline
\end{tabular}

Traits: $\mathrm{GY}=$ Grain yield, $\mathrm{FLO}_{50}=$ Days to $50 \%$ flowering, $\mathrm{PSM}_{50}=$ Days to $50 \%$ physiological maturity, $\mathrm{PRO}=$ Percentage of productive tillers, $\mathrm{PAR}=$ Panicle area

Methods: $\mathrm{Pi}=$ Cultivar superiority, $\mathrm{REML}=$ Restricted maximum likelihood, $\mathrm{YSi}=$ Yield stability index

Table 2. Correlation among stability analysis methods for grain yield

\begin{tabular}{lccccccc}
\hline Methods & Wi & $\begin{array}{c}\text { Static } \\
\text { stability }\end{array}$ & Pi & REML & ASVi & $\begin{array}{c}\text { GGE } \\
\text { biplot }\end{array}$ & YSi \\
\hline bi & -0.1 & 0.3 & -0.1 & -0.0 & -0.2 & 0.2 & -0.2 \\
Wi & 1.0 & -0.0 & 0.0 & 0.1 & -0.2 & $0.5^{*}$ & -0.2 \\
Static stability & & 1.0 & $-0.5^{*}$ & $-0.6^{*}$ & 0.3 & -0.4 & $-0.6^{*}$ \\
Pi & & 1.0 & $0.9^{* *}$ & 0.1 & $0.5^{*}$ & $0.5^{*}$ \\
REML & & & 1.0 & -0.0 & $0.5^{*}$ & $0.5^{*}$ \\
ASVi & & & & 1.0 & 0.0 & -0.3 \\
GGEbiplot & & & & & 1.0 & 0.1 \\
\hline
\end{tabular}

Methods: bi = Finlay and Wilkinson, $\mathrm{Wi}=$ Wricke's ecovalence, $\mathrm{Pi}=$ Cultivar superiority, $\mathrm{REML}$ $=$ Restricted maximum likelihood, $\mathrm{ASVi}=\mathrm{Ammi}$ stability value, $\mathrm{YSi}=$ Yield stability index

high correlation was observed between Cultivar superiority and REML, Cultivar superiority and GGE biplot, Cultivar Superiority and YSi, REML and YSi and Finley and Wilkenson and Static stability for all the traits. However, significant negative correlation was observed between Finley and Wilkenson and Static stability for most traits except grain yield.

Some consistency in genotype ranking was observed between Finley and Wilkinson and Static stability then Wricke's ecovalence, static stability and ASVi for all the traits while a similar pattern was observed between Cultivar superiority and
REML for grain yield, panicle area and percentage of productive tillers. Similarity was also observed between Wricke's ecovalence and GGE biplot for days to $50 \%$ physiological maturity and percentage of productive tillers.

\section{Grain yield (GY)}

Results of ranking of the twenty most stable genotypes for grain yield are shown in Table 3. Generally, differences in the ranking of the genotypes existed for all the seven stability analysis methods with Finley and Wilkinson, Wrike's ecovalence, static stability and ASVi identifying low 
Table 3. Genotype by environment analysis for grain yield $\left(\mathrm{kg} \mathrm{ha}^{-1}\right)$

\begin{tabular}{|c|c|c|c|c|c|c|c|c|c|c|c|c|c|c|c|c|}
\hline \multirow[t]{2}{*}{ Rank } & \multicolumn{2}{|c|}{$\begin{array}{l}\text { Finley and } \\
\text { Wilkenson }\end{array}$} & \multicolumn{2}{|c|}{$\begin{array}{l}\text { Wricke's } \\
\text { ecovalence }\end{array}$} & \multicolumn{2}{|c|}{ Static stability } & \multicolumn{2}{|c|}{$\begin{array}{l}\text { Cultivar } \\
\text { superiority }\end{array}$} & \multicolumn{2}{|c|}{ REML } & \multicolumn{2}{|c|}{ ASVi } & \multicolumn{2}{|c|}{ GGE biplot } & \multicolumn{2}{|c|}{$\begin{array}{l}\text { Yield stability } \\
\text { index }\end{array}$} \\
\hline & $\begin{array}{l}\text { Geno- } \\
\text { type }\end{array}$ & Means & $\begin{array}{l}\text { Geno- } \\
\text { type }\end{array}$ & Means & $\begin{array}{l}\text { Geno- } \\
\text { type }\end{array}$ & Means & $\begin{array}{l}\text { Geno- } \\
\text { type }\end{array}$ & Means & $\begin{array}{l}\text { Geno- } \\
\text { type }\end{array}$ & Means & $\begin{array}{l}\text { Geno- } \\
\text { type }\end{array}$ & Means & $\begin{array}{l}\text { Geno- } \\
\text { type }\end{array}$ & Means & $\begin{array}{l}\text { Geno- } \\
\text { type }\end{array}$ & Means \\
\hline 1 & $1 \mathrm{x} 8$ & 1820 & 2 & 1812 & $2 \times 12$ & 1482 & $6 \times 10$ & 2506 & $6 \times 10$ & 2324 & 2 & 1812 & $5 \times 12$ & 2322 & $3 \times 11$ & 2413 \\
\hline 2 & $1 \times 16$ & 1585 & 8 & 2005 & $6 \times 16$ & 1306 & $3 \times 11$ & 2413 & $3 \times 11$ & 2258 & $6 \times 16$ & 1306 & $6 \times 8$ & 2387 & $3 \times 12$ & 2257 \\
\hline 3 & $1 \times 9$ & 1977 & 6 & 2054 & $1 \times 16$ & 1585 & $4 \times 16$ & 2344 & $6 \times 8$ & 2210 & $1 \times 11$ & 1427 & $1 \times 14$ & 2355 & 6 & 2054 \\
\hline 4 & $4 \times 12$ & 1712 & 9 & 2027 & $4 \times 12$ & 1712 & $6 \times 8$ & 2387 & $5 \times 12$ & 2183 & $3 \times 11$ & 2413 & $5 \times 8$ & 2187 & 8 & 2005 \\
\hline 5 & $6 \times 16$ & 1306 & 12 & 1878 & $1 \times 11$ & 1427 & $3 \times 12$ & 2257 & $1 \times 14$ & 2173 & 8 & 2005 & $6 \times 7$ & 2149 & 9 & 2027 \\
\hline 6 & $2 \times 12$ & 1482 & $3 \times 9$ & 1797 & $1 \times 12$ & 1518 & $1 \times 14$ & 2355 & $6 \times 9$ & 2172 & 6 & 2054 & $4 \times 11$ & 2100 & $6 \times 10$ & 2506 \\
\hline 7 & $2 \times 15$ & 2169 & $4 \times 7$ & 1903 & 16 & 1799 & $5 \times 15$ & 2230 & $4 \times 16$ & 2171 & $2 \times 12$ & 1482 & $4 \times 14$ & 2054 & 4 & 1952 \\
\hline 8 & $4 \times 13$ & 2026 & 4 & 1952 & $5 \times 16$ & 1621 & $5 \times 12$ & 2322 & $3 \times 12$ & 2154 & $3 \times 9$ & 1797 & $5 \times 13$ & 2210 & $4 \times 16$ & 2344 \\
\hline 9 & $1 \times 7$ & 1671 & $3 \times 7$ & 1784 & 1 & 1787 & $6 \times 9$ & 2371 & $5 \times 13$ & 2102 & $4 \times 10$ & 1680 & $6 \times 11$ & 2030 & $6 \times 8$ & 2387 \\
\hline 10 & $1 \mathrm{x} 13$ & 1906 & $4 \times 10$ & 1680 & $3 \times 14$ & 1642 & $6 \times 7$ & 2149 & $5 \times 8$ & 2076 & 12 & 1878 & $2 \times 11$ & 1971 & $6 \times 7$ & 2149 \\
\hline 11 & $2 \times 7$ & 1723 & 13 & 1907 & $4 \times 7$ & 1903 & $2 \times 15$ & 2169 & $1 \times 15$ & 2071 & $4 \times 7$ & 1903 & $4 \times 8$ & 2003 & $4 \times 7$ & 1903 \\
\hline 12 & $3 \times 16$ & 1923 & 16 & 1799 & $4 \times 10$ & 1680 & $4 \times 11$ & 2100 & $6 \times 12$ & 2057 & $3 \times 12$ & 2257 & $6 \times 12$ & 2229 & 2 & 1812 \\
\hline 13 & $6 \times 14$ & 2003 & $2 \times 9$ & 1822 & 7 & 1869 & $5 \times 8$ & 2187 & $5 \times 15$ & 2046 & 9 & 2027 & $3 \times 11$ & 2413 & 12 & 1878 \\
\hline 14 & $3 \times 14$ & 1642 & 3 & 1864 & 4 & 1952 & 6 & 2054 & $4 \times 11$ & 2041 & 4 & 1952 & $1 \times 16$ & 1585 & 5 & 1993 \\
\hline 15 & $4 \times 15$ & 1821 & $3 \times 12$ & 2257 & $4 \times 15$ & 1821 & 9 & 2027 & $6 \times 7$ & 2023 & $3 \times 7$ & 1784 & $6 \times 10$ & 2506 & $1 \times 15$ & 2027 \\
\hline 16 & $5 \times 15$ & 2230 & 1 & 1787 & 2 & 1812 & $6 \times 14$ & 2003 & $2 \times 15$ & 2011 & $3 \times 13$ & 1572 & $5 \times 10$ & 1938 & 15 & 1965 \\
\hline 17 & $1 \times 12$ & 1518 & 10 & 1855 & 14 & 1922 & 8 & 2005 & $5 \times 9$ & 2002 & 16 & 1799 & 11 & 1929 & 13 & 1907 \\
\hline 18 & $6 \times 13$ & 1914 & 7 & 1869 & $3 \times 13$ & 1572 & $4 \times 14$ & 2054 & 6 & 1992 & $3 \times 10$ & 1463 & $4 \times 9$ & 1904 & $5 \times 12$ & 2322 \\
\hline 19 & $1 \times 11$ & 1427 & 14 & 1922 & $3 \times 10$ & 1463 & 15 & 1965 & $4 \times 14$ & 1988 & $1 \times 12$ & 1518 & 5 & 1984 & $4 \times 11$ & 2100 \\
\hline 20 & $5 \times 16$ & 1621 & 15 & 1965 & $3 \times 9$ & 1797 & $5 \times 13$ & 2210 & $4 \times 8$ & 1976 & 1 & 1787 & 14 & 1917 & 14 & 1922 \\
\hline
\end{tabular}


Table 4. Genotype by environment analysis for days to $50 \%$ flowering

\begin{tabular}{|c|c|c|c|c|c|c|c|c|c|c|c|c|c|c|c|c|}
\hline \multirow[t]{2}{*}{ Rank } & \multicolumn{2}{|c|}{$\begin{array}{l}\text { Finley and } \\
\text { Wilkenson }\end{array}$} & \multicolumn{2}{|c|}{$\begin{array}{l}\text { Wricke's } \\
\text { ecovalence }\end{array}$} & \multicolumn{2}{|c|}{ Static stability } & \multicolumn{2}{|c|}{$\begin{array}{l}\text { Cultivar } \\
\text { superiority }\end{array}$} & \multicolumn{2}{|c|}{ REML } & \multicolumn{2}{|c|}{ ASVi } & \multicolumn{2}{|c|}{ GGE biplot } & \multicolumn{2}{|c|}{$\begin{array}{l}\text { Yield stability } \\
\text { index }\end{array}$} \\
\hline & $\begin{array}{l}\text { Geno- } \\
\text { type }\end{array}$ & Means & $\begin{array}{l}\text { Geno- } \\
\text { type }\end{array}$ & Means & $\begin{array}{l}\text { Geno- } \\
\text { type }\end{array}$ & Means & $\begin{array}{l}\text { Geno- } \\
\text { type }\end{array}$ & Means & $\begin{array}{l}\text { Geno- } \\
\text { type }\end{array}$ & Means & $\begin{array}{l}\text { Geno- } \\
\text { type }\end{array}$ & Means & $\begin{array}{l}\text { Geno- } \\
\text { type }\end{array}$ & Means & $\begin{array}{l}\text { Geno- } \\
\text { type }\end{array}$ & Means \\
\hline 1 & $5 \times 13$ & 57.5 & 12 & 56.4 & $4 \times 14$ & 55.9 & $5 \times 7$ & 62.8 & $2 \times 11$ & 53.1 & $2 \times 14$ & 54.6 & $6 x 8$ & 59.9 & $2 \times 14$ & 54.6 \\
\hline 2 & $4 \times 14$ & 55.9 & 10 & 57.7 & 13 & 57.3 & $4 \times 15$ & 61.4 & $1 \times 9$ & 53.6 & $2 \times 16$ & 56.5 & $4 \times 13$ & 60.3 & 11 & 55.8 \\
\hline 3 & $1 \times 13$ & 58.0 & 11 & 55.8 & $5 \times 10$ & 58.3 & $3 \times 16$ & 60.4 & $1 \mathrm{x} 11$ & 54.6 & 12 & 56.4 & $4 \times 16$ & 59.9 & $2 \times 10$ & 55.6 \\
\hline 4 & $2 \times 12$ & 55.5 & $1 \times 7$ & 56.3 & $4 \times 12$ & 59.4 & $4 \times 8$ & 60.9 & $3 \times 12$ & 54.6 & $1 \times 7$ & 56.3 & $4 \times 10$ & 60.6 & $1 \times 7$ & 56.3 \\
\hline 5 & $4 \times 10$ & 60.6 & 6 & 57.5 & $6 \times 14$ & 55.6 & $6 \times 8$ & 59.9 & $2 \times 12$ & 54.9 & $4 \times 11$ & 57.5 & $4 \times 8$ & 60.9 & $1 \times 10$ & 54.8 \\
\hline 6 & $3 \times 10$ & 56.3 & 4 & 58.6 & $2 \times 7$ & 58.6 & $4 \times 10$ & 60.6 & $2 \times 14$ & 54.9 & 10 & 57.7 & $1 \times 16$ & 60.0 & 12 & 56.4 \\
\hline 7 & $5 \times 9$ & 55.8 & $4 \times 11$ & 57.5 & 10 & 57.7 & $4 \times 13$ & 60.3 & $6 \times 13$ & 55.0 & 13 & 57.3 & $1 \times 14$ & 58.4 & $2 \times 16$ & 56.5 \\
\hline 8 & $4 \times 12$ & 59.4 & $2 \times 16$ & 56.5 & $5 \times 9$ & 55.8 & $2 \times 15$ & 59.1 & $1 \times 10$ & 55.1 & 8 & 57.5 & $6 \times 10$ & 58.4 & $6 \times 14$ & 55.6 \\
\hline 9 & $3 \times 11$ & 56.1 & 7 & 58.2 & 12 & 56.4 & $1 \times 16$ & 60.0 & $2 \times 9$ & 55.6 & 11 & 55.8 & $5 \times 16$ & 57.9 & $5 \times 12$ & 55.9 \\
\hline 10 & $5 \times 10$ & 58.3 & $6 \times 16$ & 57.6 & $3 \times 7$ & 56.5 & $6 \times 15$ & 59.8 & $3 \times 8$ & 55.7 & 4 & 58.6 & 11 & 55.3 & $6 \times 12$ & 56.6 \\
\hline 11 & $3 \times 13$ & 55.9 & 1 & 57.8 & $3 \times 14$ & 58.9 & $3 \times 14$ & 58.9 & $2 \times 10$ & 55.8 & $6 \times 12$ & 56.6 & $1 \times 15$ & 58.0 & 13 & 57.3 \\
\hline 12 & $5 \times 7$ & 62.8 & 8 & 57.5 & $1 \times 10$ & 54.8 & $1 \times 12$ & 58.6 & $6 \times 14$ & 55.8 & 6 & 57.5 & $2 \times 15$ & 57.5 & $4 \times 11$ & 57.5 \\
\hline 13 & $1 \times 12$ & 58.6 & 16 & 58.5 & 11 & 55.8 & $4 \times 16$ & 59.9 & $5 \times 8$ & 56.0 & $1 \times 16$ & 60.0 & $1 \times 12$ & 58.6 & $2 \times 9$ & 54.8 \\
\hline 14 & 13 & 57.3 & $2 \times 10$ & 55.6 & 9 & 57.2 & $4 \times 12$ & 59.4 & $6 \times 11$ & 56.0 & $2 \times 10$ & 55.6 & $5 \times 13$ & 57.5 & 3 & 56.3 \\
\hline 15 & $5 \times 11$ & 56.1 & $1 \times 10$ & 54.8 & 1 & 57.8 & $3 \times 9$ & 57.9 & 11 & 56.0 & 16 & 58.5 & $6 \times 9$ & 57.6 & $3 \times 12$ & 54.1 \\
\hline 16 & $4 \times 13$ & 60.3 & 13 & 57.3 & $5 \times 11$ & 56.1 & $2 \times 7$ & 58.6 & $4 \times 14$ & 56.0 & $6 \times 16$ & 57.6 & 16 & 58.7 & $5 \times 8$ & 55.4 \\
\hline 17 & $4 \times 15$ & 61.4 & $5 \times 12$ & 55.9 & $6 \times 9$ & 57.6 & $6 \times 10$ & 58.4 & $5 \times 12$ & 56.1 & $2 \times 7$ & 58.6 & $3 \times 14$ & 58.9 & 6 & 57.5 \\
\hline 18 & $6 \times 13$ & 56.4 & $6 \times 14$ & 55.6 & $3 \times 13$ & 55.9 & $4 \times 7$ & 60.9 & $3 \times 7$ & 56.2 & 7 & 58.2 & 14 & 56.6 & 8 & 57.5 \\
\hline 19 & $3 \times 7$ & 56.5 & $6 \times 11$ & 56.5 & $6 \times 16$ & 57.6 & 16 & 58.5 & $1 \mathrm{x} 13$ & 56.2 & 1 & 57.8 & $1 \mathrm{x} 8$ & 57.4 & $4 \times 14$ & 55.9 \\
\hline 20 & $6 \times 14$ & 55.6 & $1 \times 15$ & 58.0 & $3 \times 11$ & 56.1 & 15 & 58.3 & $1 \times 7$ & 56.2 & $6 \times 14$ & 55.6 & $2 \times 11$ & 51.6 & $4 \times 9$ & 56.1 \\
\hline
\end{tabular}

1 = ICMV3771, 2 = Manganara, $3=$ Okashana2, 4 = ITMV8001, $5=$ SDMV94001, $6=$ Shibe, $7=$ Exbornu, $8=$ CIVT9206, $9=$ GGB8735, $10=$ ICMV221,

11 = ICMV221 1 white,$\quad 12$ = KatPM1, 13 = Okoa, 14 = SDMV96053, $15=$ Sosank, $16=$ Okollo 
Table 5. Genotype by environment analysis for days to $50 \%$ physiological maturity

\begin{tabular}{|c|c|c|c|c|c|c|c|c|c|c|c|c|c|c|c|c|}
\hline \multirow[t]{2}{*}{ Rank } & \multicolumn{2}{|c|}{$\begin{array}{l}\text { Finley and } \\
\text { Wilkenson }\end{array}$} & \multicolumn{2}{|c|}{$\begin{array}{l}\text { Wricke's } \\
\text { ecovalence }\end{array}$} & \multicolumn{2}{|c|}{ Static stability } & \multicolumn{2}{|c|}{$\begin{array}{l}\text { Cultivar } \\
\text { superiority }\end{array}$} & \multicolumn{2}{|c|}{ REML } & \multicolumn{2}{|c|}{ ASVi } & \multicolumn{2}{|c|}{ GGE biplot } & \multicolumn{2}{|c|}{$\begin{array}{l}\text { Yield stability } \\
\text { index }\end{array}$} \\
\hline & $\begin{array}{l}\text { Geno- } \\
\text { type }\end{array}$ & Means & $\begin{array}{l}\text { Geno- } \\
\text { type }\end{array}$ & Means & $\begin{array}{l}\text { Geno- } \\
\text { type }\end{array}$ & Means & $\begin{array}{l}\text { Geno- } \\
\text { type }\end{array}$ & Means & $\begin{array}{l}\text { Geno- } \\
\text { type }\end{array}$ & Means & $\begin{array}{l}\text { Geno- } \\
\text { type }\end{array}$ & Means & $\begin{array}{l}\text { Geno- } \\
\text { type }\end{array}$ & Means & $\begin{array}{l}\text { Geno- } \\
\text { type }\end{array}$ & Means \\
\hline 1 & $1 \times 13$ & 84.3 & 12 & 86.2 & $4 \times 14$ & 86.3 & $4 \times 16$ & 95.3 & $2 \times 11$ & 82.0 & 13 & 87.7 & $4 \times 10$ & 91.3 & $2 \times 9$ & 81.6 \\
\hline 2 & $5 \times 13$ & 88.6 & $4 \times 11$ & 87.1 & $3 \times 7$ & 87.4 & $4 \times 7$ & 94.5 & $2 \times 9$ & 82.2 & 12 & 86.2 & 4 & 90.9 & $1 \times 10$ & 83.5 \\
\hline 3 & $5 \times 10$ & 89.5 & 6 & 87.5 & $6 \times 15$ & 89.6 & $4 \times 15$ & 92.8 & $3 \times 12$ & 82.8 & 6 & 87.5 & 3 & 87.2 & $6 \times 14$ & 83.4 \\
\hline 4 & $3 \times 14$ & 89.8 & 11 & 85.2 & $1 \times 10$ & 83.5 & $3 \times 16$ & 91.5 & $1 \times 9$ & 83.0 & $4 \times 11$ & 87.1 & $2 \times 12$ & 84.4 & 11 & 85.2 \\
\hline 5 & $4 \times 14$ & 86.3 & $5 \times 11$ & 83.5 & $5 \times 10$ & 89.5 & $5 \times 7$ & 93.0 & $1 \times 13$ & 83.0 & 11 & 85.2 & $4 \times 12$ & 91.0 & 12 & 86.2 \\
\hline 6 & $6 \times 16$ & 87.4 & 15 & 88.2 & $6 \times 14$ & 83.4 & $4 \times 12$ & 91.0 & $6 \times 14$ & 83.2 & $3 \times 8$ & 86.6 & $5 \times 12$ & 84.9 & $5 \times 11$ & 83.5 \\
\hline 7 & $6 \times 9$ & 88.0 & 16 & 90.1 & $6 \times 16$ & 87.4 & $4 \times 8$ & 91.9 & $1 \times 11$ & 83.3 & 8 & 88.0 & 12 & 86.8 & $2 \times 11$ & 81.5 \\
\hline 8 & $3 \times 7$ & 87.4 & 1 & 87.7 & $6 \times 9$ & 88.0 & $4 \times 10$ & 91.3 & $5 \times 11$ & 83.4 & $1 \times 10$ & 83.5 & 11 & 84.3 & $3 \mathrm{x} 8$ & 86.6 \\
\hline 9 & $6 \times 15$ & 89.6 & 10 & 87.9 & 10 & 87.9 & $6 x 8$ & 91.5 & $1 \times 10$ & 83.5 & $1 \times 16$ & 90.4 & $3 \times 12$ & 83.0 & $2 \times 10$ & 85.0 \\
\hline 10 & $1 \times 10$ & 83.5 & $4 \times 12$ & 91.0 & 13 & 87.7 & $4 \times 13$ & 91.5 & $2 \times 12$ & 83.5 & $2 \times 7$ & 88.4 & $3 \times 14$ & 89.8 & $4 \times 11$ & 87.1 \\
\hline 11 & $5 \times 7$ & 93.0 & $2 \times 16$ & 88.3 & $3 \times 11$ & 84.3 & 16 & 90.1 & $3 \times 11$ & 84.0 & $6 \times 14$ & 83.4 & 16 & 90.3 & $4 \times 14$ & 86.3 \\
\hline 12 & $2 \times 12$ & 84.4 & $6 \times 12$ & 86.5 & 9 & 88.0 & $1 \times 16$ & 90.4 & $5 \times 8$ & 84.3 & $2 \times 9$ & 81.6 & $4 \times 11$ & 87.1 & 6 & 87.5 \\
\hline 13 & $5 \times 9$ & 89.5 & 4 & 90.0 & $1 \times 13$ & 84.3 & 4 & 90.0 & $2 \times 10$ & 84.4 & $1 \times 15$ & 87.6 & 6 & 87.6 & $2 \times 14$ & 84.1 \\
\hline 14 & $6 \times 14$ & 83.4 & $6 \times 10$ & 88.4 & $2 \times 12$ & 84.4 & $6 \times 15$ & 89.6 & $2 \times 14$ & 84.7 & $1 \times 14$ & 89.6 & $4 \times 9$ & 87.3 & 13 & 87.7 \\
\hline 15 & $1 \times 14$ & 89.6 & 13 & 87.69 & $5 \times 11$ & 83.5 & $1 \times 12$ & 90.3 & 11 & 84.9 & 10 & 87.9 & $3 \times 15$ & 88.4 & $6 \times 12$ & 86.5 \\
\hline 16 & $4 \times 15$ & 92.8 & 7 & 89.0 & 1 & 87.7 & 7 & 89.0 & $5 \times 12$ & 84.9 & $5 \times 11$ & 83.5 & 9 & 86.4 & $3 \times 11$ & 84.3 \\
\hline 17 & $4 \times 16$ & 95.3 & $5 \times 12$ & 84.9 & $3 \times 13$ & 85.1 & $5 \times 10$ & 89.5 & $3 \times 13$ & 85.0 & 15 & 88.2 & 15 & 89.0 & $5 \times 12$ & 84.9 \\
\hline 18 & $3 \times 11$ & 84.3 & $6 \times 14$ & 83.4 & 6 & 87.5 & $1 \times 8$ & 90.3 & $4 \times 14$ & 85.8 & $4 \times 14$ & 86.3 & $5 \times 11$ & 83.5 & $2 \times 8$ & 87.0 \\
\hline 19 & 13 & 87.7 & 3 & 86.5 & $3 \times 14$ & 89.8 & $3 \times 14$ & 89.8 & $6 \times 7$ & 85.9 & $2 \times 10$ & 85.0 & $5 \times 8$ & 83.6 & 8 & 88.0 \\
\hline 20 & $1 \times 12$ & 90.3 & 2 & 86.7 & 14 & 87.5 & $5 \times 9$ & 89.5 & $2 \times 8$ & 85.9 & $2 \times 8$ & 87.0 & $2 \times 11$ & 81.5 & $5 \times 15$ & 87.0 \\
\hline
\end{tabular}


Table 6. Genotype by environment analysis for percentage of productive tillers

\begin{tabular}{|c|c|c|c|c|c|c|c|c|c|c|c|c|c|c|c|c|}
\hline \multirow[t]{2}{*}{ Rank } & \multicolumn{2}{|c|}{$\begin{array}{l}\text { Finley and } \\
\text { Wilkenson }\end{array}$} & \multicolumn{2}{|c|}{$\begin{array}{l}\text { Wricke's } \\
\text { ecovalence }\end{array}$} & \multicolumn{2}{|c|}{ Static stability } & \multicolumn{2}{|c|}{$\begin{array}{l}\text { Cultivar } \\
\text { superiority }\end{array}$} & \multicolumn{2}{|c|}{ REML } & \multicolumn{2}{|c|}{ ASVi } & \multicolumn{2}{|c|}{ GGE biplot } & \multicolumn{2}{|c|}{$\begin{array}{l}\text { Yield stability } \\
\text { index }\end{array}$} \\
\hline & $\begin{array}{l}\text { Geno- } \\
\text { type }\end{array}$ & Means & $\begin{array}{l}\text { Geno- } \\
\text { type }\end{array}$ & Means & $\begin{array}{l}\text { Geno- } \\
\text { type }\end{array}$ & Means & $\begin{array}{l}\text { Geno- } \\
\text { type }\end{array}$ & Means & $\begin{array}{l}\text { Geno- } \\
\text { type }\end{array}$ & Means & $\begin{array}{l}\text { Geno- } \\
\text { type }\end{array}$ & Means & $\begin{array}{l}\text { Geno- } \\
\text { type }\end{array}$ & Means & $\begin{array}{l}\text { Geno- } \\
\text { type }\end{array}$ & Means \\
\hline 1 & $2 \times 15$ & 71.04 & 1 & 82.35 & $1 \times 9$ & 92.49 & $1 \times 9$ & 92.49 & $1 \times 9$ & 92.16 & 11 & 85.61 & 3 & 82.22 & $1 \times 9$ & 92.49 \\
\hline 2 & $1 \times 14$ & 68.5 & 14 & 82.68 & $5 \times 8$ & 91.24 & $5 \times 12$ & 91.92 & $6 \times 7$ & 91.28 & 1 & 82.35 & $4 \times 13$ & 86.76 & $1 \times 13$ & 89.46 \\
\hline 3 & $6 \times 10$ & 86.24 & 5 & 83.53 & $5 \times 12$ & 91.92 & $2 \times 11$ & 91.23 & $5 \times 8$ & 91.16 & $3 \times 12$ & 86.35 & 2 & 82.15 & $4 \times 7$ & 89.94 \\
\hline 4 & $6 \times 11$ & 88.51 & 2 & 81.69 & $2 \times 11$ & 91.23 & $6 \times 7$ & 92.17 & $5 \times 12$ & 90.98 & 14 & 82.68 & 6 & 84.21 & $4 \times 10$ & 90.56 \\
\hline 5 & $5 \times 9$ & 78.96 & 13 & 87.24 & $5 \times 10$ & 81.55 & $5 \times 8$ & 91.24 & $2 \times 11$ & 90.84 & $6 \times 15$ & 73.77 & 15 & 76.61 & $4 \times 9$ & 88.91 \\
\hline 6 & $6 \times 12$ & 84.27 & 6 & 82.65 & 9 & 82.26 & $4 \times 9$ & 88.91 & $4 \times 10$ & 90.8 & 5 & 83.53 & $1 \times 10$ & 85.93 & $4 \times 14$ & 89.79 \\
\hline 7 & $5 \times 10$ & 81.55 & 11 & 85.61 & 6 & 82.65 & $4 \times 7$ & 89.94 & $1 \times 13$ & 89.72 & 7 & 85.19 & 10 & 84.64 & 11 & 85.61 \\
\hline 8 & $1 \times 9$ & 92.49 & 10 & 82.99 & $6 \times 15$ & 73.77 & $4 \times 14$ & 89.79 & $4 \times 11$ & 89.36 & 2 & 81.69 & $5 \times 13$ & 84.82 & 7 & 85.19 \\
\hline 9 & $3 \times 13$ & 86.57 & 4 & 85.5 & $2 \times 16$ & 82.53 & 13 & 87.24 & $5 \times 7$ & 89.3 & 10 & 82.99 & $3 \times 13$ & 86.58 & $1 \times 10$ & 85.93 \\
\hline 10 & $5 \times 12$ & 91.92 & 9 & 82.26 & 5 & 83.53 & $6 \times 14$ & 87.72 & $4 \times 7$ & 89.24 & 13 & 87.24 & $2 \times 15$ & 81.48 & $4 \times 11$ & 89.19 \\
\hline 11 & $1 \times 16$ & 86.13 & $4 \times 16$ & 78.88 & 1 & 82.35 & $1 \times 13$ & 89.46 & $4 \times 14$ & 88.46 & $5 \times 8$ & 91.24 & $5 \times 16$ & 76.6 & $1 \times 16$ & 86.13 \\
\hline 12 & $5 \times 8$ & 91.24 & $2 \times 11$ & 91.23 & 12 & 82.5 & $2 \times 13$ & 87.98 & $4 \times 9$ & 88.41 & 6 & 82.65 & $2 \times 16$ & 83.39 & $4 \times 13$ & 86.75 \\
\hline 13 & $4 \times 14$ & 89.79 & $2 \times 16$ & 82.53 & $3 \times 12$ & 86.35 & $3 \times 12$ & 86.35 & $2 \times 14$ & 87.54 & $4 \times 7$ & 89.94 & 12 & 82.1 & 10 & 82.99 \\
\hline 14 & $4 \times 15$ & 74.8 & 7 & 85.19 & $5 \times 9$ & 78.96 & $6 \times 11$ & 88.52 & $3 \times 11$ & 87.53 & $1 \times 11$ & 84.23 & $6 \times 12$ & 84.27 & $2 \times 11$ & 91.23 \\
\hline 15 & $3 \times 12$ & 86.35 & $4 \times 9$ & 88.91 & $4 \times 9$ & 88.91 & 11 & 85.61 & $6 \times 8$ & 87.38 & 4 & 85.5 & 9 & 85.03 & 4 & 85.5 \\
\hline 16 & $6 \times 14$ & 87.72 & 12 & 82.5 & 10 & 82.99 & $4 \times 11$ & 89.19 & $5 \times 14$ & 87.18 & 9 & 82.26 & $2 x 8$ & 81.38 & $2 \times 13$ & 87.98 \\
\hline 17 & $2 \times 13$ & 87.98 & $1 \times 13$ & 89.46 & 14 & 82.68 & $1 \times 16$ & 86.13 & 13 & 87.15 & $2 \times 11$ & 91.23 & $5 \times 15$ & 84.62 & 9 & 82.26 \\
\hline 18 & $2 \times 12$ & 75.51 & $2 \times 9$ & 81.66 & $6 \times 14$ & 87.72 & $4 \times 13$ & 86.75 & $6 \times 11$ & 87.08 & $4 \times 16$ & 78.88 & $6 \times 16$ & 77.71 & $1 \times 11$ & 84.23 \\
\hline 19 & $6 \times 15$ & 73.77 & 3 & 82.97 & $6 \times 7$ & 92.17 & 4 & 85.5 & $4 \times 13$ & 87.08 & $4 \times 9$ & 88.91 & $3 \times 10$ & 81.81 & 1 & 82.35 \\
\hline 20 & $5 \times 11$ & 75.14 & $6 \times 7$ & 92.17 & $6 \times 11$ & 88.52 & $2 \times 14$ & 86.97 & $6 \times 14$ & 86.85 & $2 \times 16$ & 82.53 & $2 \times 9$ & 81.66 & $2 \times 14$ & 86.97 \\
\hline
\end{tabular}

1= ICMV3771, 2 = Manganara, 3 = Okashana2, 4 = ITMV8001, 5 = SDMV94001, $6=$ Shibe, 7 = Exbornu, $8=$ CIVT9206, $9=$ GGB8735, 10 = ICMV221,

$11=$ ICMV221 white, $12=$ KatPM1, $13=$ Okoa, $14=$ SDMV96053, $15=$ Sosank, $16=$ Okollo 
Table 7. Genotype by environment analysis for percentage of productive tillers

\begin{tabular}{|c|c|c|c|c|c|c|c|c|c|c|c|c|c|c|c|c|}
\hline \multirow[t]{2}{*}{ Rank } & \multicolumn{2}{|c|}{$\begin{array}{l}\text { Finley and } \\
\text { Wilkenson }\end{array}$} & \multicolumn{2}{|c|}{$\begin{array}{l}\text { Wricke's } \\
\text { ecovalence }\end{array}$} & \multicolumn{2}{|c|}{ Static stability } & \multicolumn{2}{|c|}{$\begin{array}{l}\text { Cultivar } \\
\text { superiority }\end{array}$} & \multicolumn{2}{|c|}{ REML } & \multicolumn{2}{|c|}{ ASVi } & \multicolumn{2}{|c|}{ GGE biplot } & \multicolumn{2}{|c|}{$\begin{array}{l}\text { Yield stability } \\
\text { index }\end{array}$} \\
\hline & $\begin{array}{l}\text { Geno- } \\
\text { type }\end{array}$ & Means & $\begin{array}{l}\text { Geno- } \\
\text { type }\end{array}$ & Means & $\begin{array}{l}\text { Geno- } \\
\text { type }\end{array}$ & Means & $\begin{array}{l}\text { Geno- } \\
\text { type }\end{array}$ & Means & $\begin{array}{l}\text { Geno- } \\
\text { type }\end{array}$ & Means & $\begin{array}{l}\text { Geno- } \\
\text { type }\end{array}$ & Means & $\begin{array}{l}\text { Geno- } \\
\text { type }\end{array}$ & Means & $\begin{array}{l}\text { Geno- } \\
\text { type }\end{array}$ & Means \\
\hline 1 & $2 \times 15$ & 71.0 & 1 & 82.4 & $1 \times 9$ & 92.5 & $1 \times 9$ & 92.5 & $1 \times 9$ & 92.2 & 11 & 85.6 & 3 & 82.2 & $1 \times 9$ & 92.5 \\
\hline 2 & $1 \times 14$ & 68.5 & 14 & 82.7 & $5 \times 8$ & 91.2 & $5 \times 12$ & 91.9 & $6 \times 7$ & 91.3 & 1 & 82.4 & $4 \times 13$ & 86.8 & $1 \times 13$ & 89.5 \\
\hline 3 & $6 \times 10$ & 86.2 & 5 & 83.5 & $5 \times 12$ & 91.9 & $2 \times 11$ & 91.2 & $5 \times 8$ & 91.2 & $3 \times 12$ & 86.4 & 2 & 82.2 & $4 \times 7$ & 89.9 \\
\hline 4 & $6 \times 11$ & 88.5 & 2 & 81.7 & $2 \times 11$ & 91.2 & $6 \times 7$ & 92.2 & $5 \times 12$ & 91.0 & 14 & 82.7 & 6 & 84.2 & $4 \times 10$ & 90.6 \\
\hline 5 & $5 \times 9$ & 79.0 & 13 & 87.2 & $5 \times 10$ & 81.6 & $5 \times 8$ & 91.2 & $2 \times 11$ & 90.8 & $6 \times 15$ & 73.8 & 15 & 76.6 & $4 \times 9$ & 88.9 \\
\hline 6 & $6 \times 12$ & 84.3 & 6 & 82.7 & 9 & 82.3 & $4 \times 9$ & 88.9 & $4 \times 10$ & 90.8 & 5 & 83.5 & $1 \times 10$ & 85.9 & $4 \times 14$ & 89.8 \\
\hline 7 & $5 \times 10$ & 81.6 & 11 & 85.6 & 6 & 82.7 & $4 \times 7$ & 89.9 & $1 \times 13$ & 89.7 & 7 & 85.2 & 10 & 84.6 & 11 & 85.6 \\
\hline 8 & $1 \times 9$ & 92.5 & 10 & 83.0 & $6 \times 15$ & 73.8 & $4 \times 14$ & 89.8 & $4 \times 11$ & 89.4 & 2 & 81.7 & $5 \times 13$ & 84.8 & 7 & 85.2 \\
\hline 9 & $3 \times 13$ & 86.8 & 4 & 85.5 & $2 \times 16$ & 82.5 & 13 & 87.2 & $5 \times 7$ & 89.3 & 10 & 83.0 & $3 \times 13$ & 86.6 & $1 \times 10$ & 85.9 \\
\hline 10 & $5 \times 12$ & 91.9 & 9 & 82.3 & 5 & 83.5 & $6 \times 14$ & 87.7 & $4 \times 7$ & 89.2 & 13 & 87.2 & $2 \times 15$ & 81.5 & $4 \times 11$ & 89.2 \\
\hline 11 & $1 \times 16$ & 86.1 & $4 \times 16$ & 78.9 & 1 & 82.4 & $1 \times 13$ & 89.5 & $4 \times 14$ & 88.5 & $5 \times 8$ & 91.2 & $5 \times 16$ & 76.6 & $1 \times 16$ & 86.1 \\
\hline 12 & $5 \times 8$ & 91.2 & $2 \times 11$ & 91.2 & 12 & 82.5 & $2 \times 13$ & 88.0 & $4 \times 9$ & 88.4 & 6 & 82.7 & $2 \times 16$ & 83.4 & $4 \times 13$ & 86.8 \\
\hline 13 & $4 \times 14$ & 89.8 & $2 \times 16$ & 82.5 & $3 \times 12$ & 86.4 & $3 \times 12$ & 86.4 & $2 \times 14$ & 87.5 & $4 \times 7$ & 89.9 & 12 & 82.1 & 10 & 83.0 \\
\hline 14 & $4 \times 15$ & 74.8 & 7 & 85.2 & $5 \times 9$ & 79.0 & $6 \times 11$ & 88.5 & $3 \times 11$ & 87.5 & $1 \times 11$ & 84.2 & $6 \times 12$ & 84.3 & $2 \times 11$ & 91.2 \\
\hline 15 & $3 \times 12$ & 86.4 & $4 \times 9$ & 88.9 & $4 \times 9$ & 88.9 & 11 & 85.6 & $6 \times 8$ & 87.4 & 4 & 85.5 & 9 & 85.0 & 4 & 85.5 \\
\hline 16 & $6 \times 14$ & 87.7 & 12 & 82.5 & 10 & 83.0 & $4 \times 11$ & 89.2 & $5 \times 14$ & 87.2 & 9 & 82.3 & $2 \times 8$ & 81.4 & $2 \times 13$ & 88.0 \\
\hline 17 & $2 \times 13$ & 88.0 & $1 \times 13$ & 89.5 & 14 & 82.7 & $1 \times 16$ & 86.1 & 13 & 87.2 & $2 \times 11$ & 91.2 & $5 \times 15$ & 84.6 & 9 & 82.3 \\
\hline 18 & $2 \times 12$ & 75.5 & $2 \times 9$ & 81.7 & $6 \times 14$ & 87.7 & $4 \times 13$ & 86.8 & $6 \times 11$ & 87.1 & $4 \times 16$ & 78.9 & $6 \times 16$ & 77.7 & 1x11 & 84.3 \\
\hline 19 & $6 \times 15$ & 73.8 & 3 & 83.0 & $6 \times 7$ & 92.2 & 4 & 85.5 & $4 \times 13$ & 87.1 & $4 \times 9$ & 88.9 & $3 \times 10$ & 81.8 & 1 & 82.4 \\
\hline 20 & $5 \times 11$ & 75.1 & $6 \times 7$ & 92.2 & $6 x 11$ & 88.5 & $2 \times 14$ & 87.0 & $6 \times 14$ & 86.9 & $2 \times 16$ & 82.5 & $2 \times 9$ & 81.7 & $2 \times 14$ & 876.0 \\
\hline
\end{tabular}


Table 8. Genotype by environment analysis for panicle area

\begin{tabular}{|c|c|c|c|c|c|c|c|c|c|c|c|c|c|c|c|c|}
\hline \multirow[t]{2}{*}{ Rank } & \multicolumn{2}{|c|}{$\begin{array}{l}\text { Finley and } \\
\text { Wilkenson }\end{array}$} & \multicolumn{2}{|c|}{$\begin{array}{l}\text { Wricke's } \\
\text { ecovalence }\end{array}$} & \multicolumn{2}{|c|}{ Static stability } & \multicolumn{2}{|c|}{$\begin{array}{l}\text { Cultivar } \\
\text { superiority }\end{array}$} & \multicolumn{2}{|c|}{ REML } & \multicolumn{2}{|c|}{ ASVi } & \multicolumn{2}{|c|}{ GGE biplot } & \multicolumn{2}{|c|}{$\begin{array}{l}\text { Yield stability } \\
\text { index }\end{array}$} \\
\hline & $\begin{array}{l}\text { Geno- } \\
\text { type }\end{array}$ & Means & $\begin{array}{l}\text { Geno- } \\
\text { type }\end{array}$ & Means & $\begin{array}{l}\text { Geno- } \\
\text { type }\end{array}$ & Means & $\begin{array}{l}\text { Geno- } \\
\text { type }\end{array}$ & Means & $\begin{array}{l}\text { Geno- } \\
\text { type }\end{array}$ & Means & $\begin{array}{l}\text { Geno- } \\
\text { type }\end{array}$ & Means & $\begin{array}{l}\text { Geno- } \\
\text { type }\end{array}$ & Means & $\begin{array}{l}\text { Geno- } \\
\text { type }\end{array}$ & Means \\
\hline 1 & $4 \times 12$ & 759.8 & 6 & 572.3 & $4 \times 7$ & 406.2 & $3 \times 15$ & 1065.3 & $2 \times 15$ & 1103.5 & $4 \times 9$ & 516.1 & 4 & 536.5 & $1 \times 16$ & 663.7 \\
\hline 2 & $3 \times 15$ & 1065.3 & 12 & 608.2 & $4 \times 11$ & 379.8 & $2 \mathrm{x} 8$ & 754.3 & $4 \times 15$ & 1093.9 & 6 & 572.3 & $5 \times 12$ & 770.2 & $1 \times 13$ & 654.7 \\
\hline 3 & $4 \times 7$ & 406.2 & $5 \times 14$ & 430.0 & $4 \times 16$ & 408.0 & $3 \times 10$ & 794.4 & $6 \times 15$ & 956.0 & $5 \times 14$ & 430.0 & $2 \times 7$ & 642.2 & $4 \times 12$ & 759.8 \\
\hline 4 & $2 \times 8$ & 754.3 & $4 \times 9$ & 516.1 & 4 & 533.2 & $6 \times 8$ & 718.5 & $6 \times 10$ & 942.7 & 12 & 608.2 & $4 \times 9$ & 516.1 & 10 & 600.0 \\
\hline 5 & $6 \times 8$ & 718.5 & $3 \times 9$ & 434.9 & $2 \times 9$ & 654.7 & $4 \times 12$ & 759.8 & $5 \times 12$ & 835.1 & $5 \times 11$ & 485.8 & 9 & 499.8 & 9 & 597.3 \\
\hline 6 & $2 \times 9$ & 654.7 & $1 \times 10$ & 437.1 & $6 \times 14$ & 547.2 & 15 & 655.5 & $6 \times 16$ & 759.4 & $5 \times 10$ & 418.1 & 14 & 515.9 & $4 \times 15$ & 749.5 \\
\hline 7 & $4 \times 16$ & 408.0 & $3 \times 12$ & 390.4 & $2 \times 11$ & 513.1 & $6 \times 15$ & 809.2 & 9 & 757.9 & $3 \times 9$ & 434.9 & $6 \times 16$ & 598.3 & $1 \mathrm{x} 11$ & 635.2 \\
\hline 8 & $4 \times 11$ & 379.8 & $5 \times 10$ & 418.1 & $5 \times 11$ & 485.8 & 3 & 603.3 & $6 \times 12$ & 744.5 & $6 \times 11$ & 362.6 & $4 \times 11$ & 379.8 & 8 & 563.9 \\
\hline 9 & $6 \times 14$ & 547.2 & 2 & 598.4 & $6 \times 11$ & 362.6 & 8 & 563.9 & $2 \times 9$ & 729.5 & $1 \times 10$ & 437.1 & $4 \times 16$ & 408.0 & $2 \times 8$ & 754.3 \\
\hline 10 & $2 \times 11$ & 513.1 & $6 \times 11$ & 362.6 & $2 \times 12$ & 472.6 & $6 \times 10$ & 812.9 & $6 \times 8$ & 716.3 & 10 & 600.0 & $6 \times 12$ & 634.5 & $4 \times 13$ & 643.2 \\
\hline 11 & 4 & 533.2 & $6 \times 9$ & 436.1 & 11 & 477.5 & 12 & 608.2 & $1 \times 11$ & 710.5 & $3 \times 12$ & 390.4 & $4 \times 14$ & 468.7 & 7 & 551.6 \\
\hline 12 & $2 \times 15$ & 728.3 & $6 \times 13$ & 508.6 & $6 \times 7$ & 562.7 & 10 & 600.0 & $5 \times 11$ & 674.1 & 2 & 598.4 & 15 & 734.6 & $1 \times 12$ & 579.1 \\
\hline 13 & $6 \times 7$ & 562.7 & $1 \times 15$ & 446.2 & $6 \times 12$ & 634.5 & $1 \times 16$ & 663.7 & $3 \times 15$ & 673.8 & $6 \times 9$ & 436.1 & $1 \times 7$ & 547.3 & $2 \times 10$ & 656.1 \\
\hline 14 & $4 \times 15$ & 749.5 & 10 & 600.0 & $6 \times 8$ & 718.5 & $4 \times 8$ & 526 & 16 & 633.0 & $6 \times 13$ & 508.6 & $2 \times 15$ & 749.3 & $2 \times 15$ & 728.3 \\
\hline 15 & $5 \times 11$ & 485.8 & 11 & 477.5 & $6 \times 16$ & 598.3 & $4 \times 15$ & 749.5 & 12 & 610.2 & 11 & 477.5 & $3 \times 11$ & 591.0 & $3 \times 10$ & 794.4 \\
\hline 16 & $2 \times 12$ & 472.6 & $3 \times 7$ & 491.2 & 12 & 608.2 & $2 \times 7$ & 642.2 & $2 \times 7$ & 602.3 & $1 \times 15$ & 446.2 & $1 \times 11$ & 635.2 & $2 \times 9$ & 654.7 \\
\hline 17 & $6 \times 16$ & 598.3 & 14 & 538.8 & 9 & 597.3 & 2 & 598.4 & 15 & 601.7 & $6 \times 16$ & 598.3 & $2 \times 16$ & 595.9 & $1 \times 7$ & 547.3 \\
\hline 18 & $6 \times 11$ & 362.6 & $3 \times 16$ & 452.6 & 2 & 598.4 & $6 \times 12$ & 634.5 & $1 \times 13$ & 593.0 & 16 & 562.5 & $6 \times 15$ & 809.2 & $2 \times 7$ & 642.2 \\
\hline 19 & $5 \times 7$ & 623.9 & $3 \times 8$ & 470.4 & $6 \times 13$ & 508.6 & $5 \times 12$ & 770.2 & $1 \times 16$ & 591.4 & $3 \times 8$ & 470.4 & 13 & 576.1 & 1 & 537.7 \\
\hline 20 & 9 & 597.3 & 5 & 526.9 & 6 & 572.3 & $2 \times 15$ & 728.3 & 2 & 590.1 & $3 \times 7$ & 491.2 & $2 \times 13$ & 483.9 & $3 \times 15$ & 1065.3 \\
\hline
\end{tabular}

1 = ICMV3771, 2 = Manganara, $3=$ Okashana2, 4 = ITMV8001, 5 = SDMV94001, $6=$ Shibe, 7 = Exbornu, $8=$ CIVT9206, $9=$ GGB8735, $10=$ ICMV221,

$11=$ ICMV221 white, $12=$ KatPM1, $13=$ Okoa, $14=$ SDMV96053, $15=$ Sosank, $16=$ Okollo 
yielding $\left(<2000 \mathrm{~kg} \mathrm{ha}^{-1}\right)$ genotypes as being the most stable across environments; while Cultivar superiority, REML, GGE biplot and YSi identified high yielding genotypes as being the most stable. A significant positive correlation was also observed between Cultivar superiority, REML, GGE biplot and YSi although the correlation was stronger between Cultivar superiority and REML where both methods identified 16 genotypes as being stable but with a slight difference in ranking. The Wricke's ecovalence, static stability and ASVi identified 11 out 20 genotypes as being stable although ranked differently.

\section{Days to $50 \%$ flowering $\left(\mathrm{FLO}_{50}\right)$}

The ranking of the genotypes by the methods was different for the trait, with similarity existing only in number of genotypes identified by each method (Table 4). The Finley and Wilkinson and Static stability had 10 genotypes in common, 6 with Cultivar superiority and REML while Wricke's ecovalence and ASVi had 16 in common, 8 with static stability and 7 with REML. Cultivar superiority also had 9 genotypes in common with GGEbiplot and no genotype in common with REML.

\section{Days to $50 \%$ physiological maturity $\left(\right.$ PSM $\left._{50}\right)$}

Variation in genotypes and ranking was also observed across the methods for days to $50 \%$ physiological maturity. In addition, the similarity level in number of genotypes commonly identified also varied (Table 5). The Finley and Wilkinson and Static stability methods had the highest number (13) of genotypes in common but ranked differently. This was followed by Wricke's ecovalence and GGE biplot (11), then Wricke's ecovalence and ASVi (9). The cultivar superiority had no genotype in common with REML while it had only one with ASVi.

Percentage of productive tillers (PRO)

Differences in genotypes and ranking by the stability methods were observed for productive tillers (Table 7). The Cultivar superiority and REML identified 15 of the 20 genotypes in common and 9 out of 20 with Finley and Wilkinson's while Wricke's ecovalence and ASVi had 14 of 20 most stable genotypes in common but differences existed in ranking. Using static stability, 6 out of 20 genotypes were common with REML while for GGE biplot, 6 genotypes ranked in common with Wricke's ecovalence method. The ranking for all the genotypes was different in all the stability methods tested irrespective of the commonality observed.

\section{Panicle area (PAR)}

Variation in ranking of the most stable genotypes by the tested stability methods was also observed for panicle area although some similarities among the methods existed (Table 8 ). The Finley and Wilkinson and static stability had 14 genotypes in common of the 20 most stable; while Cultivar stability and REML methods identified 12 genotypes in common. In addition, Wricke's ecovalence and ASVi identified 17 common genotypes out of 20 most stable genotypes across environments. The GGE biplot identified 6 common genotypes as Cultivar superiority and REML while 5 common genotypes were identified by Finley and Wilkinson and Static stability.

\section{Discussion}

Across the evaluation sites, yield ranged between $1427 \mathrm{~kg} \mathrm{ha}^{-1}$ to $2506 \mathrm{~kg} \mathrm{ha}^{-1}$. The ANOVA indicated significant variation 
among the genotypes tested and the GEI, showing that the multiplicative interaction of the genotypes and environments affected the performance of the test materials as also reported by Subi et al. (2013). However, as noted by Crossa (1990), ANOVA does not explore the underlying structure within the GEI and thus other methods were adapted. Significant correlation among the Cultivar superiority with REML, YSi and GGE biplot shows that a prediction of comparable results can be revealed when any of the methods is used independently with minimal variation in the ranking of the genotypes.

Significant correlation was also observed elsewhere between Cultivar superiority and $\mathrm{YSi}$ in cotton (Blanche Sr., 2005) and Faba bean (Temesgena et al., 2015) studies. These correlated methods aid in simultaneously selecting stable and high yielding genotypes unlike the Finlay and Wilkinson, Wricke's ecovalence, ASVi and Static stability which, in this study, identified mostly low yielding genotypes as being the most stable. Except ASVi, similar observations were made by Mohammadi and Amri (2008) in studies on wheat. Wrike's method has also been reported to identify low yielding genotypes in sugar cane (Mendes de Paula et al., 2014) and field pea (Fikere et al., 2014) as also observed in this study.

The various analysis methods ranked genotypes differently for the same traits across the test environments. Similar observations were also made by Pabale and Pandya (2010) when they compared Eberhart and Russell (1966), Perkins and Jinks (1968) and Freeman and Perkins (1971) models in ranking of pearl millet genotypes basing on grain yield. Mustapha and Bakari (2014) reported no similarity between static and cultivar superiority; while cultivar superiority and GGE biplot identified the same genotypes as being stable, but ranked them differently in pearl millet. In this study, Cultivar superiority and GGE biplot were significantly correlated for grain yield, days to 50\% flowering and days to $50 \%$ physiological maturity; with a difference in ranking of genotypes. Variation in ranking of genotypes was also reported by Parmar et al. (2012) when they compared nonparametric tests in rice; Mosleh et al. (2012) when they compared Wricke's ecovalence, Shukla stability variance, rank test, and Eberhart and Russell; and Namorato et al. (2009) when they compared AMMI and Eberhart and Russell methods in maize. The inconsistency in ranking was also reported by Alberts (2004) and Khosa (2012) when cultivar superiority, Finlay and Wilkinson, Wricke's ecovalence and ASVi were compared in maize. In addition, Dehghani et al. (2008) also observed variation in ranking Lentil genotypes, although they observed similarity between Shukla and Wricke's, cultivar superiority and Wricke's ecovalence, Finlay and Wilkinson and cultivar superiority. However, in the present study the methods had no significant correlation. The lack of significant association and differential ranking of genotypes by ASVi and GGE biplot was also observed in wheat studies by Naroui Rad et al. (2013). Results showed no significant association between cultivar superiority and Finlay and Wilkinson's methods as also reported by Purchase et al. (2000). On the contrary, Purchase et al. (2000) reported a significant correlation between ASVi and Wricke's ecovalence as also noted by Alberts (2004). This implies that results from the comparisons may greatly depend on the method, types of genotypes and 
environments being evaluated as also observed by Westcott (1986) and thus more than one method should be used to characterise and explore performance of genotypes across environments as also suggested by Lin and Binns (1988).

\section{Acknowledgement}

The National Semi Arid Resources Research Institute for financial support and the technical staff who helped with trial management and data collection.

\section{References}

Alberts, M.J.A. 2004. A comparison of statistical methods to describe genotypexenvironment interaction and yield stability in multi-location maize trials. MSc. Thesis, University of Free State, Bloemfontein, South Africa.

Bartlett, M.S. 1937. Properties of Sufficiency and Statistical Tests. Proceedings of the Royal Society A: Mathematical, Physical and Engineering Sciences 160 (901):268.

Bashir, E.M.A., Ali, A.M., Adam Ali, M., Ismail, M.I., Parzies, H.K. and Haussmann, B.I.G. 2014. Patterns of pearl millet genotype-by-environment interaction for yield performance and grain iron $(\mathrm{Fe})$ and zinc $(\mathrm{Zn})$ concentrations in Sudan. Field Crops Research 166:82-91.

Becker, H.C. and Léon, J. 1988. Stability analysis in plant breeding. Plant Breeding 101:1-23.

Blanche, Sr. S.B. 2005. New methods to assess cotton varietal stability and identify discriminating environments. PhD Thesis, Louisiana State University.
Crossa, J. 1990. Statistical analysis of multilocational trials. Advances in Agronomy 44:55-85.

Dehghani, H., Sabaghpour, S.H. and Sabaghnia, N. 2008. Genotype x environment interaction for grain yield of some lentil genotypes and relationship among univariate stability statistics. Spanish Journal of Agricultural Research 6(3):385-394. Eberhart, S.A. and Russell, W.A. 1966. Stability parameters for comparing varieties. Crop Science 6:36-40.

Falconer, D.S. 1990. Selection in different environments: effects on environmental sensitivity (reaction norm) and on mean performance. Genetics Research Cambridge 56:57-70.

Farshadfar, E., Mahmodi, N. and Yaghotipoor, A. 2011. AMMI stability value and simultaneous estimation of yield and yield stability in bread wheat (Triticum aestivum L.). Australian Journal of Crop Science 5(13):18371844.

Francis, T.R. and Kannenberg, L.W. 1978. Yield stability studies in shortseason maize. 1. Adescriptive method for grouping genotypes. Canadian Journal of Plant Science 58:10291034.

Freeman, G.H. and Perkins, J.M. 1971. Environmental and genotypeenvironmental components of variability VIII. Relations between genotypes grown in different environments and measures of these environments. Heredity 27:15-23.

Fikere, M., Bing, D.J., Tadesse, T. and Ayana, A. 2014. Comparison of biometrical methods to describe yield stability in field pea (Pisum sativum L.) under southeastern Ethiopian 
conditions. African Journal for Agriculture Research 9 (33):25742583.

Finlay, K.W. and Wilkinson, G.N. 1963. The analysis of adaptation in a plant breeding programme. Australian Journal of Agricultural Research 14:742-754.

Gauch, H.G. 1988. Model selection and validation for yield trials with interaction. Biometrics 44:705-715.

Gauch, H.G. 2006. Statistical analysis of yield trials by AMMI and GGE. Crop Science 46:1488-1500.

Gauch, H.G., Piepho, H.P. and Annicchiarico, P. 2008. Statistical analysis of yield trials by AMMI and GGE: Further considerations. Crop Science 48:866-889.

Gebre, W. 2014. Evaluation of pearl millet (Pennisetum glaucum L.) genotypes for yield and yield stability in South Omo and West Hararghe. Journal of Biology, Agriculture and Healthcare 4(8):99-121.

Gruneberg, W.J., Manrique, K., Zhang, D. and Hermann, M. 2005. Genotype x environment interactions for a diverse set of sweet potato clones evaluated across varying eco-graphic conditions in Peru. Crop Science 45:2160-2171.

IBPGR and ICRISAT. 1993. Descriptors for pearl millet [Pennisetum glaucum (L.) R. Br.]. International Board for Plant Genetic Resources, Rome, Italy; International Crops Research Institute for the Semi-Arid Tropics, Patancheru, India.

IFAD. 1999. Farmer participatory testing of technologies to increase sorghum and pearl millet production in the Sahel. www.ifad.org/grants.

Integrated Breeding Platform's Breeding Management System version 3.0.8 (IBP-BMS). 2014. Integrated
Breeding Platform, CIMMYT, MexicoVeracruz.

Ishaq, J. and Meseka, S. 2014. Genetic Stability of Grain Yield and principal component analysis in pearl millet (Pennisetum glaucum L.). Greener Journal of Plant Breeding and Crop Science 2(4):88-92.

Kang, M.S. 1993. Simultaneous selection for yield and stability in crop performance trials: Consequences for growers. Agronomy Journal 85:754757.

Kaya, Y., Aksura, M. and Taner, S. 2006. GGE-Biplot analysis of multienvironment yield trials in bread wheat. Turkish Journal of Agriculture and Forestry 30:325-337.

Khairwal, I.S., Yadav S.K., Rai K.N., Upadhyaya H.D., Kachhawa D., Nirwan B., Bhattacharjee R., Rajpurohit B.S., Dangaria, C.J. and Srikant. 2007a. Evaluation and identification of promising pearl millet germplasm for grain and fodder traits. Journal of SAT Agricultural Research 5(1):1-5.

Khosa, S. 2012. Assessment of maize germplasm lines for genetic diversity, cultivar superiority and combining ability. MSc Thesis, University of KwaZulu Natal, South Africa.

Lavoranti, O.J., dos Santos Dias, C.T. and Kraznowski, W.J. 2007. Phenotypic stability via AMMI model with bootstrap re-sampling. Pesquisa Florestal Brasileira 54:45-52.

Lin, C.S. and Binns, M.R. 1988. A superiority measure of cultivar performance for cultivar $\mathrm{x}$ location data. Canadian Journal of Plant Science 68:193-198.

Mohammadi. R. and Amri, A. 2008. Comparison of parametric and nonparametric methods for selecting 
stable and adapted durum wheat genotypes in variable environments. Euphytica 159:419-432.

Mosleh, R.Z., Sepas, A.Y., Chaichi, M. and Lalehloo, B.S. 2015. The Analysis of Genotype-Environment Interactions: Comparison of Parametric and NonParametric Tests for Interactions in Bread Wheat Genotypes in Cold Regions of Iran. MAGNT Research Report 3(3):103-109.

Mustapha, M. and Bakari, H.R. 2014. Statistical Evaluation of Grain Yield in Millet Trials Using Principal Component Analysis. Current Trends in Technology and Sciences journal 3(6):422-428.

Namorato, H., Miranda, G.V., Vagno de Souza, T., Oliveira, L.R., DeLima, R.O. and Mantovani, E.E. 2009. Comparing Biplot Multivariate Analyses with Eberhart and Russell' method for genotype $\mathrm{x}$ environment interaction. Crop Breeding and Applied Biotechnology 9:299-307.

Naroui Rad, M.R., Abdul Kadir, M., Rafii, M.Y., Jaafar, H.Z.E., Naghavi, M.R. and Ahmadi F. 2013. Genotype $\times$ environment interaction by AMMI and GGE biplot analysis in three consecutive generations of wheat (Triticum aestivum) under normal and drought stress conditions. Australian Journal of Crop Science 7(7):956961.

Pabale, S.S. and Pandya, H.R. 2010. A comparison of different stability models for genotype $\mathrm{x}$ environment interaction in pearl millet. Electronic Journal of Plant Breeding 1(5):1294-1298.

Parmar, D.J., Patel, J.S., Mehta, A.M., Makwana, M.G. and Patel S.R. 2012. Non-Parametric methods for interpreting genotype $\mathrm{x}$ environment interaction of rice genotypes (Oryza
Sativa L.). Journal of Rice Research 5:1-2.

Patterson, H.D. and Thompson, R. 1971. Recovery of inter-block in formation when block sizes are unequal. Biometrika 58:545-554.

Payne, R.W., Murray, D.A., Harding, S.A., Baird, D.B. and Soutar, D.M. 2012 Genstat $^{\circledR}$ for Windows ${ }^{\mathrm{TM}}$, 15th Edition, Introduction. VSN International, Oxford.

Perkins, J.M., and Jink J.L. 1968. Environmental and genotype environmental components of variability. III. Multiple lines and crosses. Heredity 23(2):39-256.

Pinthus, M.J. 1973. Estimate of genotypic value: A proposed method. Euphytica 22:121-123.

Purchase, J.L., Hatting, H. and Van Deventer, C.S. 2000. Genotype x environment interaction of winter wheat (T. aestivum) in South Africa: Stability analysis of yield performance. South African Journal of Plant and Soil 17(3):101-107.

Rai, K.N., D.S. Murty, D.J. Andrews. and Bramel-Cox P.J. 1999. Genetic enhancement of pearl millet and sorghum for the semi-arid tropics of Asia and Africa. Genome 42:617-628. Serraj, R., Bidinger, F.R., Chauhan, Y.S., Seetharama, N., Nigam S.N. and Saxena, N.P. 2003. Management of drought in ICRISAT cereal and legume mandate crops. pp. 127-144. In: Kijne, J.W., Barker R. and Molden, D. (eds.). Water Productivity in Agriculture: Limits and Opportunities for Improvement. CAB International.

Shukla, G.K. 1972. Some statistical aspects of partitioning genotypeenvironmental components of variability. Heredity 29:237-245. 
Subi, M.I.M. and Idris, A.E. 2013. Genetic variability, heritability and genetic advance in pearl millet (Pennisetum glaucum [L.] R. Br.) genotypes. British Biotechnology Journal 3(1):54-65.

Tai, G.C.C. 1971. Genotypic stability analysis and application to potato regional trials. Crop Science 11:184190.

Temesgena, T., Kenenib, G., Seferaa, T. and Jarsob, M. 2015. Yield stability and relationships among stability parameters in faba bean (Vicia faba L.) genotypes. The Crop Journal 3:258-268.

Vadez, V., Hash, T., Bidinger, F.R. and J. Kholova. 2012. Phenotyping pearl millet for adaptation to drought. Frontiers of Physiology 3:1-12. doi: 10.3389/fphys.2012.00386.
Westcott, B. 1986. Some methods of analysing genotype-environment interaction. Heredity 56:243-253.

Yan, W. and Hunt L.A. 2002. Genetic and environmental causes of GXE interaction for winter wheat yield in Ontario. Crop Science 41:19-25.

Yan, W., M.S. Kang, Ma, B., Woods, S. and Cornelius, P.L. 2007. GGE Biplot vs. AMMI Analysis of genotype-byenvironment data. Crop Science 47(2):643-653.

Yan, W. and Racjan, I. 2002. Biplot analysis of test environments and trait relations of soybean in Ontario. Crop Science 42:11-20.

Yau, S.K. 1991. Need of scale transformation in cluster analysis of genotypes based on multi-location yield data. Journal of Genetics and Breeding 45:71-76. 